\title{
Políticas y programas de alimentación y nutrición en México
}

\author{
Simón Barquera, MD, MSC, ${ }^{(1)}$ Juan Rivera-D ommarco, MSC, PhD, ${ }^{(1)}$ \\ A lejandra G asca-García, MD (1)
}

Barquera S, Rivera-Dommarco J, Gasca-García A. Políticas y programas de alimentación y nutrición en México.

Salud Publica Mex 2001:43:464-477.

El texto completo en inglés de este artículo está disponible en: http://www.insp.mx/salud/index.html

\section{Resumen}

México tiene una larga historia de implementación de programas y políticas orientadas a mejorar la nutrición de grupos vulnerables. A pesar de ello, la desnutrición constituye uno de los retos de salud pública más importantes en México. El presente artículo proporciona una perspectiva histórica de las principales estrategias, programas y políticas que han surgido en México, analizando su diseño e implementación, así como algunos resultados obtenidos. Finalmente, se plantean puntos críticos para iniciar una discusión en torno a los problemas relacionados con la alimentación y las políticas que en México se proponen para solucionarlos. El texto completo en inglés de este artículo está disponible en: http://www.insp.mx/salud/index.html

Palabras clave: política alimentaria; programas de nutrición; México

\author{
Barquera S, Rivera-Dommarco J, Gasca-García A. \\ Food and nutrition policies \\ and programs in Mexico. \\ Salud Publica Mex 2001:43:464-477. \\ The English version of this paper \\ is available at: http://www.insp.mx/salud/index.html
}

\section{A bstract}

Mexico has a long-standing history of programs and policies oriented to improve the nutrition of vulnerable groups. However, malnutrition represents one of the main public health challenges in Mexico.This article provides a historic perspective of the main strategies, programs and policies that have been carried out in Mexico, as well as a detailed analysis of their design, implementation, and selected results. Finally, some critical issues related to food policies implemented in Mexico are identified. The English version of this paper is available at: http://www.insp.mx/salud/ index.html

Key words: food policy; nutrition programms; Mexico

(1) División de N utrición, Centro de Investigación en Salud Poblacional, Instituto N acional de Salud Pública, México.

Fecha de recibido: 2 de agosto de 2000 - Fecha de aprobado: 6 de abril de 2001

Solicitud de sobretiros: Dr. Simón Barquera. Jefe del Departamento de Dieta y Enfermedades C rónicas de la División de Nutrición y Salud. Centro de Investigación en Salud Poblacional, Instituto N acional de Salud Pública. Avenida Universidad 655, colonia Santa María A huacatitlán, 62508, Cuernavaca, Morelos, México. Correo electrónico: sbarquera@ correo.insp.mx 


\section{Cuadro I \\ Evolución DE LOS PRINCIPALES PROGRAMAS Y POLÍTICAS ALIMENTARIAS Y DE NUTRICIÓn en MÉXICO, 1922-1994}

\begin{tabular}{|c|c|c|c|c|}
\hline Año* & Política o programa & Objetivo & Población blanco & Estrategia 0 acción \\
\hline 1922-1924 & $\begin{array}{l}\text { Diver sos programas asisten- } \\
\text { ciales }^{\ddagger}\end{array}$ & A poyo a los consumidores & $\begin{array}{l}\mathrm{N} \text { iños en edad escolar de } \\
\text { zonas urbanas }\end{array}$ & Implementación de desayunos escolares \\
\hline 1925 & Subsidio a la producción & $\begin{array}{l}\text { Aumentar la producción agríco- } \\
\text { la y mejorar la producción gana- } \\
\text { dera }\end{array}$ & $\begin{array}{l}\text { Productores agrícolas y de } \\
\text { ganado }\end{array}$ & $\begin{array}{l}\text { O rganización de cooperativas po pulares, préstamo a los pro- } \\
\text { ductores, combate a plagas de la agricultura y ganadería, ex- } \\
\text { posición nacional ganadera, control de la producción lechera }\end{array}$ \\
\hline 1936-1937 & $\begin{array}{l}\text { Almacenes } \mathrm{N} \text { acionales de } \\
\text { Depósito§ } \\
\text { Comité Regulador del Mer- } \\
\text { cado de Trigo } \\
\text { Comité Regulador del Mer- } \\
\text { cado de Subsistencias Po- } \\
\text { pulares }\end{array}$ & $\begin{array}{l}\text { Controlar el precio de granos en } \\
\text { el mercado } \\
\text { Mejorar el acceso a los artículos } \\
\text { de consumo de primera necesi- } \\
\text { dad }\end{array}$ & Población de bajos recursos & $\begin{array}{l}\text { Almacenamiento de grano para regulación de precios en el } \\
\text { mercado } \\
\text { Regulación del mercado de subsistencia, adaptación de la } \\
\text { economía a las necesidades sociales, importación de maíz y } \\
\text { trigo }\end{array}$ \\
\hline 1940 & $\begin{array}{l}\text { Mecanización de la agricul- } \\
\text { tura } \\
\text { Técnica de la alimentación } \\
\text { Segundo Plan Sexenal }\end{array}$ & $\begin{array}{l}\text { Mejorar la producción } \\
\text { Mejorar el estado de nutrición } \\
\text { Educar a la población }\end{array}$ & $\begin{array}{l}\text { Población económicamente } \\
\text { débil, grupos menesterosos }\end{array}$ & $\begin{array}{l}\text { Adquisición de maquinaria agrícola, crédito a la producción } \\
\text { Creación de comedores populares, cadena de expendios } \\
\text { populares de leche, sale al mercado la primera leche re- } \\
\text { hidratada } \\
\text { Fundación del Instituto N acional de N utriología }\end{array}$ \\
\hline 1942 & $\begin{array}{l}\text { Primer programa de io- } \\
\text { dación de la sal }\end{array}$ & $\begin{array}{l}\text { Disminuir enfermedades por de- } \\
\text { ficiencia de iodo }\end{array}$ & $\begin{array}{l}\text { Toda la población, principal- } \\
\text { mente la de comunidades } \\
\text { con bocio endémico }\end{array}$ & lodación y distribución de la sal \\
\hline $1946-1950$ & $\begin{array}{l}\text { Abasto popular } \\
\text { Subsidio a la producción de } \\
\text { alimentos }\end{array}$ & $\begin{array}{l}\text { A baratar el costo de artículos ali- } \\
\text { mentarios en el mercado } \\
\text { Mejorar la producción }\end{array}$ & Población de bajos recursos & $\begin{array}{l}\text { Importación de artículos escasos en el país, control de pre- } \\
\text { cios de artículos de primera necesidad,tecnificación del cam- } \\
\text { po, ganadería, creación de la Comisión N acional de Leche, } \\
\text { desayunos escolares. Revolución verde (semilla mejorada) }\end{array}$ \\
\hline 1958 & $\begin{array}{l}\text { Abasto y subsistencias po- } \\
\text { pulares } \\
\text { Plan de acción inmediata } \\
\text { Procuraduría Federal de De- } \\
\text { fensa al Consumidor }\end{array}$ & $\begin{array}{l}\text { Regular precios de los artículos } \\
\text { básicos } \\
\text { A poyo a la producción } \\
\text { Protección a la economía popu- } \\
\text { lar }\end{array}$ & Población de bajos recursos & $\begin{array}{l}\text { Control de precios de los artículos básicos } \\
\text { Precios de garantía a la producción, mejorar el almacenaje y } \\
\text { distribución de productos agrícolas } \\
\text { Vigilancia y dirección de la economía nacional }\end{array}$ \\
\hline 1961-1965 & $\begin{array}{l}\text { Subsidio al consumo de pro- } \\
\text { ductos del campo }\end{array}$ & $\begin{array}{l}\text { Incrementar el ingreso con pre- } \\
\text { cios de garantía para productos } \\
\text { del campo }\end{array}$ & $\begin{array}{l}\text { Población rural y de bajos } \\
\text { recursos }\end{array}$ & $\begin{array}{l}\text { Creación de Conasuposa y la Compañía Rehidratadora de } \\
\text { Leche } \\
\text { Mantener reservas de productos de primera necesidad } \\
\text { Regular precios de productos de primera necesidad en el } \\
\text { mercado }\end{array}$ \\
\hline 1972 & Subsidio al consumo & $\begin{array}{l}\text { Proteger la economía y mejorar } \\
\text { el poder adquisitivo, regular pre- } \\
\text { cios en el mercado }\end{array}$ & Población de bajos recursos & $\begin{array}{l}\text { Compras reguladoras de maíz y frijol,transformación de Co- } \\
\text { nasuposa a Leche Industrializada Conasupo (Liconsa) }\end{array}$ \\
\hline $1975-1980$ & $\begin{array}{l}\text { Sistema N acional para el } \\
\text { Programa de Apoyo al Co- } \\
\text { mercio Ejidal (PACE) } \\
\text { Desarrollo Integral de la Fa- } \\
\text { milia (DIF) } \\
\text { SistemaAlimentario Mexica- } \\
\text { no (SAM) }\end{array}$ & $\begin{array}{l}\text { Educar a la población en hábitos } \\
\text { de alimentación y distribución de } \\
\text { complementos dietéticos } \\
\text { Ampliación de la red comercial } \\
\text { Subsidiar a la producción y me- } \\
\text { jorar el estado nutricional de la } \\
\text { población }\end{array}$ & $\begin{array}{l}\text { Población infantil } \\
\text { Madres gestantes }\end{array}$ & $\begin{array}{l}\text { Desayunos escolares, fabricación de leche para lactantes y } \\
\text { madres gestantes, creación de cocinas populares, distribución } \\
\text { de semillas y hortalizas } \\
\text { Crédito para la producción de semillas, producción y comer- } \\
\text { cialización de fertilizantes, subsidio a la producción y dis- } \\
\text { tribución de alimentos } \\
\text { Regulación de los precios de los productos de consumo }\end{array}$ \\
\hline 1982 & $\begin{array}{l}\text { Programa Nacional de Ali- } \\
\text { mentación (Pronal) } \\
\text { Pacto de Solidaridad } \\
\text { Económica }\end{array}$ & $\begin{array}{l}\text { A poyar la producción, dis- } \\
\text { tribución y consumo de alimen- } \\
\text { tos, cambiar condiciones de } \\
\text { alimentación y nutrición }\end{array}$ & $\begin{array}{l}\text { Población con alto índice de } \\
\text { marginación, menores de } \\
\text { cinco años, mujeres embara- } \\
\text { zadas y lactantes }\end{array}$ & $\begin{array}{l}\text { Incremento de salarios mínimos sobre la canasta básica, fo- } \\
\text { mento a la producción de alimentos, programas de vigilancia } \\
\text { del estado nutricional, orientación alimentaria }\end{array}$ \\
\hline 1990 & $\begin{array}{l}\text { Programa de Educación, Sa- } \\
\text { lud y Alimentación (Pro- } \\
\text { gresa) }\end{array}$ & $\begin{array}{l}\text { Mejorar los niveles de salud y nu- } \\
\text { trición }\end{array}$ & $\begin{array}{l}\text { Población de bajos ingresos, } \\
\text { niños menores de cinco } \\
\text { años y mujeres embarazadas }\end{array}$ & $\begin{array}{l}\text { Vigilancia del estado de salud y nutrición, distribución de su- } \\
\text { plemento alimentario, educación en salud y nutrición }\end{array}$ \\
\hline 1994 & $\begin{array}{l}\text { Programa de suplemen- } \\
\text { tación con megadosis de vi- } \\
\text { tamina } A\end{array}$ & $\begin{array}{l}\text { Proteger contra deficiencia de vi- } \\
\text { tamina A }\end{array}$ & $\begin{array}{l}\mathrm{N} \text { iños menores de cinco } \\
\text { años }\end{array}$ & $\begin{array}{l}\text { Dar suplementación a los niños durante las campañas de va- } \\
\text { cunación }\end{array}$ \\
\hline
\end{tabular}


México. Por razones de espacio no se pretende profundizar en éstos, sino más bien describir sus principales características de diseño, la experiencia de implantación y algunos de sus resultados, poniendo atención específica en las políticas orientadas a aumentar el consumo de alimentos.

México tiene una historia importante de crisis (ambientales, sociales, económicas y políticas) con efectos en la seguridad alimentaria y la nutrición. A principios del siglo XX se produjeron epidemias, sequías y hambrunas recurrentes debido a condiciones ambientales adversas y a una pobre infraestructura para hacerles frente, lo que ocasionó muertes y emigración masiva de mexicanos a los Estados Unidos de América (EUA). ${ }^{1}$ Estas crisis también han estimulado el desarrollo de programas y políticas para preservar la seguridad alimentaria, y han servido como instrumento alterno a la reforma agraria para contener la movilización de grupos organizados que ejercen presión para la obtención de mejoras en sus condiciones de vida.,3

Los programas y políticas alimentarios en México guardan una estrecha relación con la estabilidad nacional a partir de la Revolución de 1910, motivada en parte por una fuerte demanda de repartición de tierras, que se tradujo en la adopción de una política agraria que atendió en cierta medida dicho problema. Por varias décadas el Estado procuró mantener su hegemonía y legitimidad instrumentando de forma moderada políticas redistributivas, entre las cuales se encontraron diversos proyectos de reforma agraria. Sin embargo, las medidas que se tomaron nunca fueron suficientes ni tuvieron un impacto significativo en el desarrollo de las zonas rurales de extrema pobreza; además, crearon dificultades entre el Estado y los grupos de poder locales, por lo cual, éste optó por generar mecanismos distributivos (como diversos tipos de subsidios) para mejorar el bienestar social, contener la demanda por aumentos al salario mínimo, mantener su legitimidad y al mismo tiempo evitar confrontaciones con grupos de poder. ${ }^{2}$

De los programas de distribución de alimentos, en México se han probado prácticamente todas las modalidades, desde un sistema de subsidio a los consumidores (que va de los programas de desayunos escolares a la creación de la Compañía Nacional de Subsistencias Populares Conasupo), hasta un sistema de subsidios a los productores; ${ }^{4}$ asimismo, son diversas las agencias que han estado involucradas en él (cuadro II). Sin embargo, se benefició sobre todo a poblaciones de zonas urbanas, mientras las poblaciones rurales marginales recibieron beneficios en una proporción menor. ${ }^{5}$

En México las políticas alimentarias han formado parte de los programas de desarrollo y han respondido a coyunturas de tipo ambiental, económico y político. Durante la expropiación petrolera México se vio en la necesidad de considerar la producción de granos como un punto prioritario para la seguridad nacional; en 1973 se perdió la autosuficiencia en maíz y comenzó a aumentar el déficit en la producción de otros granos (más de 25\% del maíz era importado). La producción de granos para el consumo fue una de las líneas estratégicas a las que el gobierno destinó cantidades importantes de recursos, lo cual fue posible en gran parte gracias al precio favorable del petróleo.

El Estado creó en 1980 el Sistema Alimentario Mexicano (SAM), un programa que tuvo por objeto estimular la producción de alimentos básicos (facilitando el acceso a créditos y mejorando los precios de garantía, entre otras estrategias) para alcanzar la autosuficiencia y mejorar la distribución de alimentos, sobre todo para los sectores marginales. Este programa se caracterizó por la coexistencia de diversas agencias, sectores y secretarías, y su planeación y coordinación quedó a cargo del Sistema Nacional de Evaluación, presidido por un grupo de asesores del Presidente de la República, mientras que en su desarrollo participaron tanto organizaciones gubernamentales (la Secretaría de Agricultura y Recursos Hidráulicos SARH, la Secretaría de la Reforma Agraria SRA y la Secretaría de Programación y Presupuesto SPP), como organizaciones no gubernamentales (Asociación de Banqueros Mexicanos ABM, Confederación de Cámaras Nacionales de Comercio Concanaco, etc.); cada una participó en diferentes áreas y con diferentes actividades para el desarrollo de este programa (cuadro III). Esta participación multisectorial creó dificultades entre las estructuras involucradas en el programa, principalmente entre la Secretaría de Agricultura y Recursos Hidráulicos SARH y la Secretaría de Programación y Presupuesto SPP. A pesar de un diseño aparentemente innovador, tres años después el programa fue liquidado entre otras razones debido a la fuerte crisis fiscal. ${ }^{6,7}$

A partir de 1983 se crea el Programa Nacional de Alimentación (Pronal), como una de las medidas de ajuste estructural para disminuir el gasto público reduciendo significativamente los subsidios relacionados con el consumo y producción de alimentos. Dicho plan identifica entre sus objetivos aumentar el consumo de alimentos en zonas vulnerables, eliminando la necesidad de tener una agencia coordinadora. Su ejecución, a diferencia del SAM, no creó tensiones administrativas al interior de las secretarías y agencias gubernamentales, tampoco generó elevadas pérdidas fiscales ni desperdicio de ventajas comparativas en la producción de otros bienes.

En 1985, después del temblor que afectó a la ciudad de México y algunos estados de la República, el

salud pública de méxico / vol.43, no.5, septiembre-octubre de 2001 


\section{Cuadro II \\ Principales agencias, organizaciones y empresas paraestatales relacionadas con programas Y POLÍTICAS DE ALIMENTACIÓN Y NUTRICIÓN EN MÉXICO*}

\begin{tabular}{|c|c|c|}
\hline Tipo de política & Nombre de la agencia & Descripción \\
\hline Producción & Compañía N acional de Subsistencias Populares (Conasupo) & Subsidio a la producción de alimentos \\
\hline & Banco N acional de Crédito Rural (Banrural) & Crédito a los productores \\
\hline & Fideicomiso para la Reforma Agraria (Fira) & Crédito a los productores \\
\hline & Secretaría de la Reforma Agraria (SRA) & Impulso a organización campesina \\
\hline & Confederación $\mathrm{N}$ acional Campesina & Impulso a organización campesina \\
\hline & Secretaría de Agricultura y Recursos Hidráulicos (SARH) & $\begin{array}{l}\text { Cambio tecnológico, desarrollo agroindustrial, riesgo compartido y } \\
\text { organización campesina }\end{array}$ \\
\hline & Secretaría de Hacienda y Crédito Público & Alianza y riesgo compartido \\
\hline & Productora N acional de Semillas (Pronase) & Cambio tecnológico \\
\hline & Fertilizantes Mexicanos (Fertimex) & Cambio tecnológico y ampliación de red comercial \\
\hline & Confederación de Cámaras Internacionales (Concamin) & O rganización industrial \\
\hline & Confederación de Cámaras de Comercio (Concanaco) & O rganización comercial \\
\hline & Secretaría de Comercio (Secom) & Ampliación de red comercial \\
\hline Consumo & $\begin{array}{cl}\text { Compañía N acional de Subsistencias Populares (C onasupo) } \\
\text { - } & \text { Leche Industrializada Conasupo, S.A. (Liconsa) } \\
\text { - } & \text { Distribuidora Conasupo, S.A. (Diconsa) } \\
\text { - } & \text { Maíz Industrializado Conasupo, S.A. (Minsa) } \\
\text { - } & \text { Trigo Industrializado Conasupo, S.A. (Triconsa) } \\
\text { - } & \text { Industrializadora Conasupo, S.A. (Iconsa) } \\
\text { - } & \text { Impulsora del Pequeño Comercio, S.A. (Impecsa) }\end{array}$ & $\begin{array}{l}\text { Subsidios al consumo de alimentos } \\
\text { Leche } \\
\text { Venta de alimentos al menudeo } \\
\text { Maíz } \\
\text { Pan } \\
\text { Granos y semillas } \\
\text { Venta de alimentos al mayoreo }\end{array}$ \\
\hline & Fideicomiso liquidador de subsidio a la tortilla (Fidelist) & Subsidio al precio de la tortilla \\
\hline & $\begin{array}{l}\text { Sistema N acional para el D esarrollo Integral de la Familia } \\
\text { (DIF) }\end{array}$ & Programas de alimentación y salud \\
\hline & Secretaría de Salud (SSA) & $\begin{array}{l}\text { Distribución de insumos y actividades de educación en nutrición y } \\
\text { salud }\end{array}$ \\
\hline & Secretaría de D esarrollo Social (Sedesol) & $\begin{array}{l}\text { Distribución de alimentos y programas de educación en nutrición y } \\
\text { salud }\end{array}$ \\
\hline & Secretaría de Educación Pública (SEP) & Programas de desayunos escolares \\
\hline
\end{tabular}

* Algunas de estas agencias ya no existen (como en el caso de Conasupo), y otras actualmente no se encuentran desarrollando los programas referidos

gobierno puso en marcha planes de emergencia para proteger la seguridad alimentaria de las poblaciones afectadas, en este caso con una importante participación de la sociedad civil. En México, organizaciones no gubernamentales adquirieron importancia estableciendo diversos programas asistenciales, preventivos y educativos para mejorar la alimentación del país.
En diciembre de 1987 se experimentó una importante devaluación del peso con un aumento generalizado en los precios de alimentos y otros bienes, ante lo cual el Estado convoca a un "pacto de solidaridad económica". Muchos de los programas anteriores son restructurados en diversos grados; así, a principios de los noventa se inicia el programa integral "Solida- 
Cuadro III

Principales sectores involucrados en el desarrollo del Sistema Alimentario Mexicano

Nivel de participación

Agencia

Descripción

Planeación y evaluación

Sistema N acional de Evaluación

Ejecución: producción

SARH

Asistencia técnica a los productores que recibieron crédito

Banrural, Firac

Crédito para la producción de semillas

Fertimex

Producción y comercialización de fertilizantes

Pronase

Promoción de la ampliación de la red de demanda y producción de semillas

Ejecución: comercialización y distribución Conasupo:

Diconsa

Liconsa

Minsa, etcétera

Subsidio a la producción y distribución de alimentos

Regulación de los precios de la producción

Regulación de los precios de los productos de consumo

ridad", el cual abarca varios programas dirigidos a la salud, educación, distribución de alimentos y mejoras en los servicios públicos de las comunidades. Su objetivo es elevar el nivel de salud de la población rural, urbana e indígena con altos índices de marginación. Dentro de las acciones que plantea para beneficiar a la población en materia de alimentación y nutrición se incluyen la vigilancia del estado nutricional de los niños menores de cinco años, la orientación alimentaria y el fomento a la producción de alimentos. En diciembre de 1994, de nueva cuenta, México experimentó una devaluación que fue producto de una balanza de pagos negativa, un control inadecuado del tipo de cambio, un sistema bancario débil y el retiro masivo de capital extranjero, entre las razones más importantes.

La magnitud de la crisis económica, aunada a la grave caída en los precios del petróleo, hicieron necesaria la adopción de medidas de ajuste estructural buscando una disminución importante del gasto público. Para ello el gobierno diseñó un programa social integral denominado Progresa (Programa de Educación, Salud y Alimentación) al mismo tiempo que dio fin a la existencia de Conasupo, organismo paraestatal que operaba gran parte de los subsidios generalizados en el país desde hacía casi 41 años.

A continuación se describirán los programas y políticas más importantes que se han aplicado en México. Estos han sido clasificados como políticas relacionadas con la producción y políticas relacionadas con el consumo. Para facilitar su ubicación temporal, se han ordenado en el cuadro I, donde se señalan sus principales objetivos explícitos, grupo blanco y principales estrategias.

\section{Políticas relacionadas con la producción de alimentos}

En el presente trabajo no se revisan de forma individual las políticas de fomento a la producción; sin embargo, dada su importancia crítica dentro de las políticas alimentarias y de nutrición, se mencionan las principales líneas de acción en México.

Entre los insumos para la producción con que cuentan estos programas están, en primer lugar, los relacionados con el capital y el riesgo, como el acceso a créditos, la alianza para el riesgo compartido, el precio de garantía y los subsidios a insumos (como fertilizantes, tractores, etc.); en segundo lugar están aquéllos relacionados con la mejora de la eficiencia de los sistemas, como la ampliación de la red estatal de comercialización, el impulso a la organización campesina, la asesoría y la capacitación en la toma de decisiones y el cambio tecnológico, por citar algunos ejemplos.

Las políticas que tienen como fin otorgar insumos relacionados con el capital y el riesgo pueden ser un fuerte incentivo para aumentar o disminuir la producción. Su objetivo es proteger a los campesinos contra bajas drásticas de precio y a los consumidores urbanos contra el aumento de los precios, ${ }^{5}$ tal como sucede con el subsidio del maíz, en el que la participación del Estado va desde la compra del grano a precio de garantía, hasta su participación en la producción y venta de alimentos básicos a precios subsidiados. Sin embargo, puede haber pérdidas fiscales importantes si los precios internacionales bajan demasiado. También se corre el riesgo de mantener a los productores trabajando en un mercado en el que posi- 
blemente no tengan las ventajas comparativas reales que podrían tener con otros cultivos, lo que aumentaría su vulnerabilidad más tarde, cuando los precios de garantía desaparezcan. El objetivo de promover subsidios a los productores y consumidores, otorgados por Conasupo durante años, se tradujo en un déficit fiscal para la agencia que representaba, en 1982, 49.5\% del déficit total de empresas paraestatales, con excepción de Petróleos Mexicanos (Pemex). ${ }^{5}$

Un programa creado para apoyar a los campesinos en la venta de sus cosechas fue el Programa de Apoyo al Comercio Ejidal (1975). Su objetivo fue aumentar el poder de negociación de los campesinos en el mercado de granos. Este programa buscaba, ante todo, apoyar a grupos organizados de campesinos y no a individuos, así como también a los campesinos de áreas con mayor aislamiento comercial. Entre las principales formas de apoyo se encontraban los descuentos en transporte y la creación de centros de recepción para la venta de grano. En la práctica, este programa no enfrentó la dificultad de atender a los campesinos de zonas aisladas, y más bien se canalizó hacia los campesinos con alta productividad y que se encontraran en zonas en las que Conasupo tenía una amplia red de centros de recepción; en dichos lugares hubo problemas por falta de motivación en el personal responsable de los mismos para hacer participar a los pequeños productores, mientras los campesinos que tenían producción excedente, y los intermediarios, "compraban" el acceso rápido a los centros de recepción. Además, se sumó a estas dificultades, de acuerdo con algunos autores, la percepción que tenían los directivos del programa, según los cuales el problema de la pobreza en el campo residía en "aspectos relacionados con los conocimientos formales, el desempeño y la actitud de los campesinos", y no en la falta de recursos, la inequidad y el tipo de relaciones de poder entre los campesinos y ejidatarios.

En cuanto a los insumos para mejorar la capacidad técnica y administrativa, la organización campesina es una de las áreas que presenta mayores retos dentro de los programas dirigidos a la producción, ya que muchos campesinos, especialmente los de menores recursos, cultivan no sólo para vender sino también para subsistir, por lo que mejoras en sistemas de administración, planeación y almacenamiento se traducen directamente en el consumo y la seguridad alimentaria, y no sólo como un efecto en el aumento de la producción neta.

Las experiencias en insumos tecnológicos para mejorar la producción no han sido totalmente afortunadas, debido a la gran complejidad que representa la adopción de medidas que en ocasiones no están adecuadamente ajustadas a la realidad del campesino en regiones con perfiles ecológicos diferentes. Un ejemplo de esto es el caso del SAM, pues durante su vigencia se apoyó a pequeños agricultores con un paquete tecnológico que creó dudas entre los campesinos, por el riesgo de que las condiciones locales resultaran inadecuadas para el desarrollo de semillas con características especiales, lo que pondría en riesgo la producción de subsistencia. ${ }^{6}$ Otro problema importante que se presentó fue el de corrupción entre los sectores que otorgaban y recibían apoyo financiero; este problema resultó ser importante, ya que se refiere a cantidades cuantiosas y a grados de corrupción de gran magnitud.

\section{Políticas relacionadas con el consumo de alimentos}

A diferencia de las políticas relacionadas con la producción de alimentos, cuyos indicadores giran en torno a los bienes de consumo (distribución, volumen producido, ahorro generado, etcétera), las políticas y programas relacionados con el consumo de alimentos tienen un efecto más directo sobre el estado nutricional $^{*} \mathrm{y}$ las pautas de consumo. Es importante hacer la distinción entre subsidios a los alimentos e intervenciones directas sobre nutrición dirigidas a grupos vulnerables (como programas de alimentación complementaria, suplementación alimentaria y educación nutricional). Estas últimas representan una alternativa de mayor complejidad en su ejecución y frecuentemente su efecto suele ser menor que el esperado; 8,9 sin embargo, aun con estas desventajas, en la coyuntura internacional actual son consideradas como una opción de mayor costo-efectividad, y su éxito depende de problemáticas particulares y de un monitoreo riguroso. Por otra parte, los subsidios generalizados tienen el riesgo de corrupción, son de costo elevado y carecen de selectividad de los grupos vulnerables.

A continuación se presentan las principales modalidades de subsidio a los consumidores establecidos en México.

Conasupo. En México, por su magnitud, destacan los programas desarrollados gracias a Conasupo, con el objeto de aumentar el consumo de alimentos de los

\footnotetext{
* Aunque también se podría valorar, al evaluar un programa de subsidios, los efectos de éste a nivel microeconómico, por ejemplo, en el ingreso y gasto del hogar de pequeños productores, así como variaciones en la estructura de su gasto en alimentos y patrones de consumo.
} 
estratos más pobres del país; mediante sus diversas agencias participó principalmente con subsidios generalizados (como el del precio de la tortilla) y selectivos (como el de la distribución de leche a familias de escasos recursos). ${ }^{10}$ Durante la última fase de su existencia estuvo experimentando diversas formas de convertir los subsidios generalizados en subsidios selectivos en las zonas urbanas del país, ya que aquéllos no contribuían a mejorar la capacidad adquisitiva de los grupos más pobres, y representaban un gasto importante de recursos. ${ }^{11}$

Programas selectivos. Una alternativa para mejorar esta situación fue el diseño de criterios de inclusión para la recepción del subsidio, como el que se llevó dentro del programa de Tortivales (1990)*, los cuales incluían en un principio a los beneficiarios del programa de Tortibonos (1985-1989), la ampliación de la cobertura de este programa a otras familias con el criterio de inclusión, la elaboración de un mapa de pobreza con requisitos similares a los planteados por Conasupo para el programa de leche industrializada, y la verificación de las características de las familias que recibían el subsidio por medio de encuestas similares a las aplicadas por Leche Industrializada Conasupo, S.A. (Liconsa), y que serán descritas posteriormente. Este programa tuvo por objeto proporcionar un kilogramo de tortilla sin costo a las familias de menores ingresos de zonas urbanas, que percibían un ingreso igual o menor a dos salarios mínimos. El proceso se administró de forma novedosa, con tarjetas similares a las de crédito, que eran aceptadas en las tortillerías afiliadas, donde un lector electrónico con memoria registraba el detalle de las transacciones. Esta parte administrativa del sistema de tarjetas quedó a cargo del Banco Nacional de México (Banamex), mientras la Conasupo se encargó del monitoreo de la distribución de la tortilla.

El programa mostró su viabilidad y fue una experiencia innovadora, sin embargo, resultó sumamente complejo en su realización, ya que requirió identificar constantemente a las poblaciones vulnerables con estudios socioeconómicos y geográficos; además, requería evaluaciones periódicas de su funcionamiento y supervisión permanente a los industriales involucrados con todos los insumos del programa, y nutricionalmente sólo aumentó el consumo de maíz en las poblaciones urbanas. Este programa y el de leche in-

\footnotetext{
* El programa de Tortivales iniciado en 1990 tiene su antecedente en el programa de los Tortibonos, que operó de 1985 a 1989. Estos últimos no tuvieron una cobertura extensiva y su selectividad no era muy confiable, por lo que con apoyo del Banco Mundial el programa fue rediseñado en 1990.
}

dustrializada fueron criticados, además, por no incluir en sus estrategias un componente educativo.

Programa de cupones para leche de Liconsa. En 1972 se crea Liconsa, derivada de una rama de Conasupo (la Compañía Rehidratadora de Leche, 1965), con el objetivo principal de proveer a familias con ingreso menor a dos salarios mínimos, con una transferencia de ingreso en forma de leche a bajo costo, asegurando cierto consumo de ésta para mejorar el estado nutricional de los niños, aunque más tarde se incluyeron también las mujeres embarazadas y en lactancia, así como los ancianos. Los beneficiarios fueron seleccionados mediante estudios socioeconómicos y geográficos; se creó un mapa de pobreza que clasificó las zonas geográficas en microrregiones según su estado de desnutrición (de acuerdo con siete indicadores) y marginalidad (de acuerdo con 19 indicadores). ${ }^{1,12} \mathrm{La}$ cantidad se determinaba cuando la familia entraba al programa, (se proveyó a cada familia con $570 \mathrm{ml}$ de leche por día, cantidad que se aumentó por cada niño o persona en la familia con las características de inclusión). A diferencia de otros programas, la distribución de leche de Liconsa se consideró innovadora, ya que contó con un diseño basado en la selección de grupos vulnerables, en lugar de ser un subsidio generalizado. En 1974 se introdujo al programa una tarjeta familiar para mejorar la selectividad de los beneficiarios. A lo largo de los años se han hecho otros cambios y ajustes en las áreas de administración, proceso, abastecimiento e infraestructura; en su administración intervienen varios sectores, instituciones y la comunidad. La figura 1 ejemplifica la complejidad en la coordinación de este tipo de programas.

En 1988 el programa tuvo un costo por beneficiario de aproximadamente 20 dólares anuales de EUA. En 1991, el costo aumentó a 118 dólares anuales para una familia con dos niños en el programa y cubría a 2.9 millones de familias, siendo $78.3 \%$ del Distrito Federal y del estado de México, combinadas. La cobertura del programa en 1988 fue "de 50\% de las familias urbanas con ingresos por debajo de los dos salarios mínimos"; sin embargo, su cobertura rural mostró ser limitada y por tal motivo pudo estar estimulando la urbanización. Asimismo, habría que reflexionar sobre el grado de beneficio que tiene el programa en zonas rurales, ya que en ellas la leche se distribuyó en polvo para disminuir problemas logísticos, pero con el riesgo de contaminarse fácilmente durante su rehidratación, realizada por los consumidores, y su permanencia en recipientes sin refrigeración. Desde el punto de vista nutricional contribuyó a aumentar el consumo de proteínas en las familias beneficiadas. Sin embargo, se llegó a estimar que $40 \%$ de las familias 


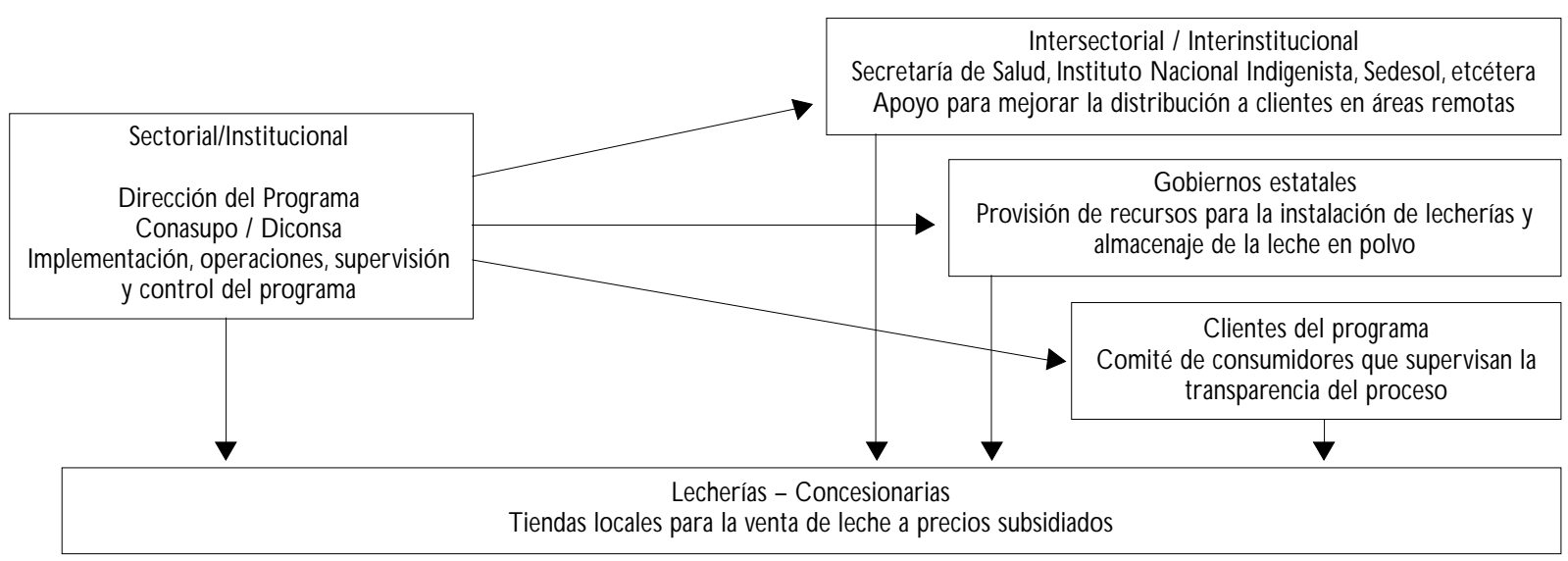

Figura 1. Niveles de coordinación en la administración de Liconsa. MéXico

que recibían el insumo tenían ingresos mayores a lo estipulado. ${ }^{11}$ Este es un ejemplo claro de un programa de subsidio selectivo y los tipos de problemas que se presentan en su operación.

Suplementación con vitamina A. En 1987 la Organización Mundial de la Salud (OMS) estimó que la deficiencia de vitamina A era endémica en 39 países; México está considerado entre los países que actualmente presenta este problema de salud pública. A partir de 1993 se llevó a cabo en México un programa de suplementación con megadosis de vitamina A como parte de las acciones que se realizan durante las Semanas Nacionales de Salud. Dicho programa se basó en evidencias presentadas por diversos estudios en los que se encontró reducción de mortalidad en niños menores de cinco años. Los beneficiarios de este programa fueron los niños en edad prescolar en zonas marginadas..$^{13}$ Desafortunadamente, no se cuenta aún con una evaluación integral que muestre el impacto de este programa en la población; sin embargo, existe una evaluación parcial que demostró la pertinencia de la suplementación con esta vitamina y la necesidad de repetirla periódicamente (de 4 a 6 meses) mientras no haya otras estrategias que permitan su obtención por medio de la dieta; asimismo, se menciona en esta evaluación la posible relación que existe entre la suplementación de vitamina A y captación de hierro en los niños. ${ }^{14}$

Programas de asistencia alimentaria del Sistema Nacional para el Desarrollo Integral de la Familia (DIF). Desde 1929 se iniciaron en México programas de atención a la niñez por parte de instituciones públicas, siguiendo diversas modalidades como desayunos escolares. ${ }^{15}$ Uno de los organismos más involucrados con la implementación de estos programas es el Sistema Nacional para el Desarrollo Integral de la Familia (DIF). Este organismo cuenta con alrededor de 10 programas, entre los cuales se encuentran el programa de raciones alimentarias (Desayunos Escolares-DIF), asistencia social alimentaria a familias, cocinas populares y unidades de servicios integrales, dotaciones gratuitas de leche en polvo (IMSS-Solidaridad, Liconsa y DIF), medicina preventiva y nutrición, y alimentación familiar, entre otros. ${ }^{16}$ Estos programas han logrado acciones integradas dirigidas a desarrollar modelos alimentarios y tienen una cobertura importante de grupos vulnerables. En 1994, tan sólo con los programas de raciones alimentarias y despensas familiares se cubrió una población de 1.7 y 8.8 millones, respectivamente, de personas (DIF, Instituto Nacional Indigenista -INI-, Niños en Solidaridad); para 1998 se distribuían diariamente 4599363 desayunos en el país con dos modalidades (caliente y frío), los cuales cubrieron entre 20 y $30 \%$ de los requerimientos diarios de energía y proteínas de los grupos beneficiados; asimismo, el programa de asistencia social alimentaria benefició en ese año a 1810348 familias. ${ }^{15,17}$ En 1995 se creó un programa interinstitucional (en el que participaron las Secretarías de Desarrollo Social, Educación Pública, Salud, Agricultura, Ganadería y Desarrollo Rural, además del DIF y el Instituto Mexicano del Seguro Social, entre otras instituciones) para mejorar la nutrición y la salud de los miembros más vulnerables de la familia. Este programa se denominó Programa de Alimentación y Nutrición Familiar. Sus acciones se dirigieron a los 
menores de cinco años, prescolares y escolares, así como a las familias en zonas de extrema pobreza, zonas indígenas, zonas rurales y en zonas urbanas marginales. Se consideró importante clasificar al país en 12 regiones, por la diversidad de características de las comunidades. El programa funciona a través de tres ejes estratégicos que son: a) Canasta Básica Alimentaria, que consiste en la distribución de alimentos que cubran las necesidades esenciales de las familias indígenas y rurales; b) Programa de Desayunos Escolares, que consiste en dar a los niños prescolares y escolares un desayuno que aporte $30 \%$ de los requerimientos diarios de proteínas y calorías; c) Programa de Apoyo Alimentario en Zonas Urbanas Marginadas, que consiste en el subsidio a la tortilla y dotación de leche, y que funciona actualmente en las zonas urbanas marginales. Un aspecto sumamente relevante de los programas implementados por el DIF es que han logrado trascender a los cambios sexenales y su permanencia contrasta con otros programas establecidos en México.

Otros programas de atención a la nutrición en México. De forma paralela a los programas nacionales anteriormente mencionados, en México se han ejecutado una serie de programas dirigidos a grupos vulnerables (niños, mujeres embarazadas o en lactancia, ancianos, grupos de pobreza marginal, etcétera), que básicamente proveen una transferencia de ingreso, principalmente en forma de alimentos para aumentar el consumo. En algunos casos los programas tienen además un componente educativo. Sus criterios de selección son variables y en general no han sido suficientemente evaluados. Entre ellos se encuentran los programas integrales de la Secretaría de Salud (Programa de Nutrición y Salud) y del IMSS (IMSS-Coplamar, IMSSSolidaridad, IMSS-Progresa) para elevar el estado de salud y proteger a los grupos vulnerables mediante una serie de medidas que combinan monitoreo y vigilancia integral del estado de salud, orientación y educación en alimentación y, en algunos casos, fomento a la producción de alimentos. ${ }^{18}$

Programa de Educación, Salud y Alimentación. ${ }^{19}$ El programa nacional Progresa fue diseñado para beneficiar a poblaciones marginales de bajos ingresos. Los hogares de las localidades seleccionadas reciben un paquete de educación en salud y nutrición, así como una beca a la familia por cada uno de los niños menores de 18 años que se encuentren estudiando entre tercero de primaria y tercero de secundaria en las escuelas públicas. Junto con estas acciones, se proveen apoyos para mejorar la calidad de las escuelas públicas.
El componente de salud busca de forma integral y sinérgica mejorar la cobertura en las unidades de atención de primer nivel para disminuir la incidencia de enfermedades entre los miembros del hogar y mejorar su situación de salud. Las acciones están dirigidas a todos los beneficiarios de Progresa en el hogar y en particular a los más vulnerables: los niños menores de cinco años y mujeres embarazadas o en lactancia. Para alcanzar este objetivo fueron diseñadas tres estrategias: a) proveer el Paquete Básico de Servicios de Salud (PBSS); b) prevenir la desnutrición desde la edad prenatal, y c) promover y mejorar el cuidado a la salud y la atención en las unidades del primer nivel de atención. El componente de alimentación además incluye una transferencia de recursos a familias seleccionadas para contribuir a mejorar la cantidad y diversidad del consumo de alimentos, promoviendo así un mejor estado nutricional. La distribución de un suplemento alimentario es otro componente de Progresa dirigido a todos los niños de 4 a 23 meses de edad y a los de 24 a 59 meses con desnutrición, así como a las mujeres embarazadas, ${ }^{*}$ el cual está diseñado para proveer alrededor de $20 \%$ de los requerimientos calóricos y $100 \%$ de los de micronutrimentos.

La participación de la comunidad en el programa es vista como una parte esencial para alcanzar los objetivos del programa; sin embargo, se reduce al cumplimiento de acciones ya establecidas por el programa. Una vez que los beneficiarios son contactados se les proporciona información acerca de las obligaciones contraídas y beneficios obtenidos al ingresar.

Las instituciones gubernamentales involucradas en la planeación y ejecución del programa son varias; no incluyen sólo al sector salud, sino también al sector educativo, al de desarrollo y al administrativo; cuenta, además, con asesoría de instituciones internacionales, como el Banco Mundial. La Secretaría de Desarrollo Social, a través del Consejo Interinstitucional del Progresa (Conprogresa) es la encargada de hacer la selección y actualización de las familias beneficiarias. Esta instancia además opera el sistema de entrega de apoyos monetarios, realiza el seguimiento y la evaluación del programa. Las Secretarías de Educación Pública y de Salud son las responsables de establecer las normas y los lineamientos de operación del programa, mientras que el Consejo Nacional de Fo-

\footnotetext{
* Los suplementos están diseñados con una formulación distinta para los niños y las madres.
} 
mento Educativo y el programa IMSS-Solidaridad tienen a su cargo la operación de sus respectivos componentes. En el ámbito estatal las autoridades de salud y educación prestan los servicios a la población beneficiaria, comprueban su asistencia periódica a las actividades establecidas y verifican el cumplimiento de las responsabilidades contraídas mediante un sistema de registro. La entrega de apoyo monetario se realiza a través de las oficinas de Telecomunicaciones de México. ${ }^{20}$

Entre las críticas que ha enfrentado el Progresa está el hecho de destinar sus acciones directamente a miembros de comunidades, identificados en pobreza extrema, es decir, que forman parte de una política de salud selectiva; en este sentido el beneficio que se obtiene está fuera del alcance de muchos otros miembros con necesidades similares. Las acciones que plantea para la solución de problemas responden a indicadores de tipo económico y se realizan de acuerdo con su costo y efectividad, de manera que la salud se convierte en un bien al que se tiene acceso de acuerdo con la capacidad adquisitiva del beneficiario.

Por otro lado, se le reconoce el hecho de favorecer la permanencia de los niños en las escuelas, particularmente la de las niñas, lo cual se traduce en mejores oportunidades futuras de empleo y desarrollo para esta población.

La implementación de un programa de esta magnitud es compleja, ya que involucra diversos sectores y agencias en los ámbitos nacional, estatal, regional y local que interactúan para producir efectos sinérgicos con el fin de mejorar la educación, la salud y el estado nutricional de las poblaciones vulnerables. Además de una estructura compleja en el país, el Programa enfrenta el reto de no tener en los estados necesariamente el mismo organigrama, por lo que las acciones se tienen que interpretar y adaptar antes de su puesta en marcha en las localidades (figura 2).

Programas de fortificación con micronutrimentos en México. La fortificación de alimentos con determinados micronutrimentos es muy atractiva para los planificadores de políticas, por la sencillez de su planeación, bajo costo y resultados. ${ }^{21}$ Sin embargo, presenta problemas como la aceptación que puede tener entre la población y, en ocasiones, se requiere dar incentivos a los productores. Iodación de sal. Desde 1889 se documentó el problema de bocio endémico en México. En 1942, por decreto presidencial, el consumo de sal iodada se hizo obligatorio como estrategia para la disminución de las enfermedades por deficiencia de yodo. Dicha estrategia se llevó a cabo en las comunidades cuya prevalencia de bocio fuera mayor a 20\%; para ese entonces nueve estados de la República mexicana cumplían esta característica, incluyendo al Distrito Federal. A partir de esa fecha la regulación de la iodación de sal ha sido implementada en diversas ocasiones. A principios de los sesenta Maisterrena y colaboradores describieron una alta prevalencia de deficiencia de yodo en algunas regiones montañosas de México, sin embargo, hasta la fecha no se tiene información precisa sobre este problema en México. ${ }^{22,23}$ Los programas de iodación de sal resultaron complejos en su implementación, ya que en ella participaron varios sectores - como la entonces Secretaría de Industria y Comercio- en la regulación de la producción de sal con una adecuada iodación, la Secretaría de Salud a través de su Departamento de Control de Alimentos, Bebidas y Medicamentos que marca los lineamientos y normas para la iodación, y la Cámara de Comercio, distribuyendo y comercializando el producto. Actualmente existe una norma oficial mexicana (NOM-040-SSA1-1993) que establece las especificaciones sanitarias para la sal iodada y la sal fluorada, y se prepara otra para la prevención, tratamiento y control de los desórdenes por la deficiencia de iodo (PROY-NOM-SSA).

Enriquecimiento de harinas. A partir de 1999, en México se inició un acuerdo para fortificar las harinas de maíz nixtamalizado y de trigo, procesadas industrialmente, con micronutrimentos con el fin de restaurar las vitaminas y minerales que se pierden durante el proceso que se sigue para obtener harina refinada, en el cual se elimina hasta $70 \%$ del contenido de micronutrimentos. ${ }^{24}$ Durante el enriquecimiento también se añaden a las harinas algunos micronutrimentos que, se considera, no son aportados de manera adecuada con la dieta. Aunque esta medida no es por sí sola una solución al problema de las deficiencias, ya que no todos los mexicanos consumen alimentos procesados, es una medida que puede tener un impacto importante, puesto que la base de la dieta en el país son estos cereales y un gran número de habitantes los consumen. Sobre todo si se considera como una de varias alternativas nacionales para mejorar el estado nutricional. Entre las desventajas de este tipo de programas se puede mencionar que algunos grupos rurales no consumen tortillas elaboradas con harinas industrializadas y quedan, por lo tanto, excluidos de los beneficios que éstos aportan; además, en el caso de la población infantil, el consumo de tortillas fortificadas implica un consumo de micronutrimentos mayor al recomendado, ya que la fortificación se realiza para cubrir requerimientos de la población adulta.

Un punto importante respecto a la instrumentación de estos programas en México, como en otras 


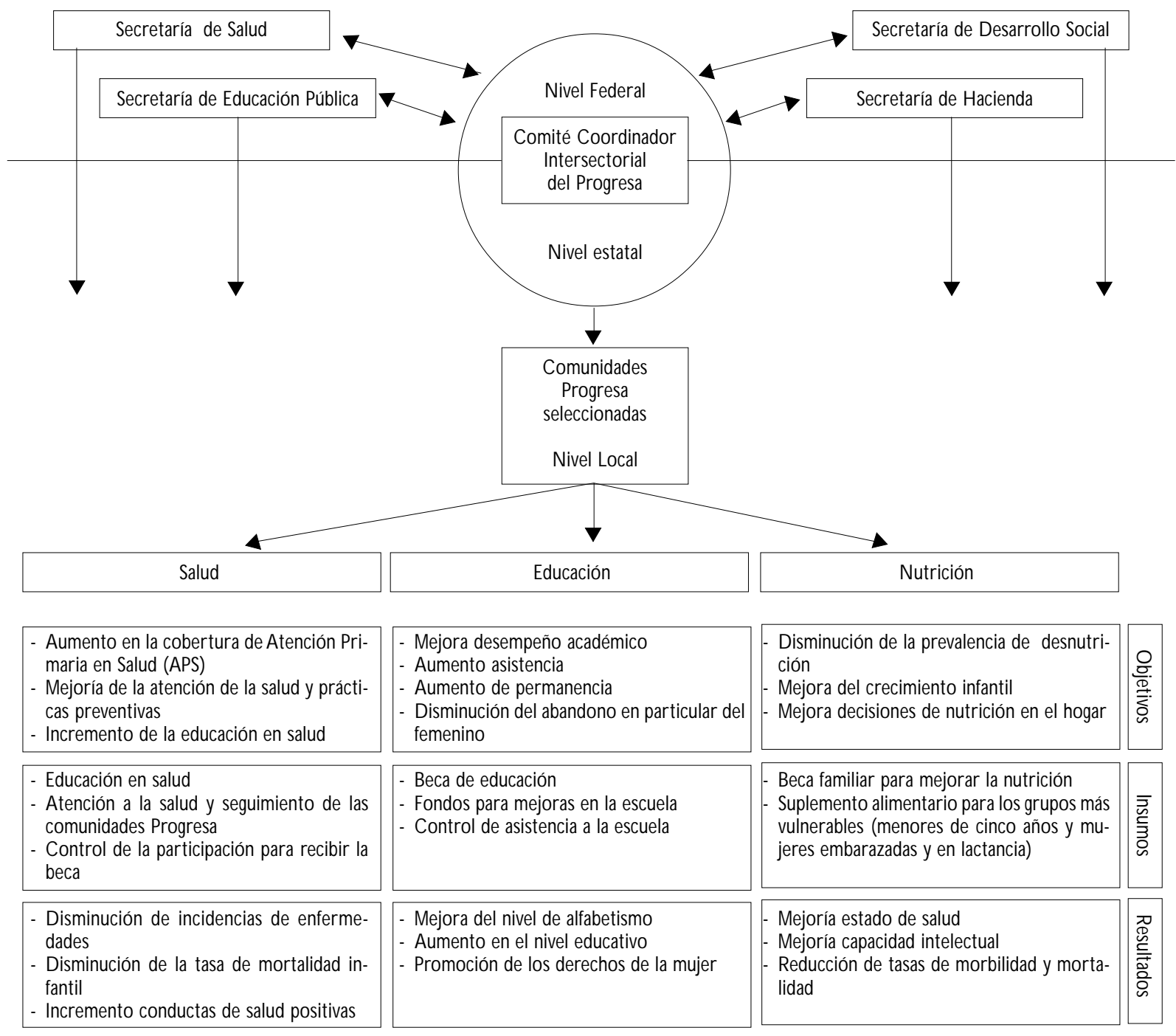

Figura 2. Esquema del Programa de Educación, Salud y Alimentación. México

partes del mundo, es que el papel del Estado es de regulador y promotor, y no de proveedor, ya que el costo de los insumos, en este caso, es cubierto por los productores y por la población a través de su consumo, debido a que éstos sufren sólo una ligera elevación del costo.

\section{Conclusiones}

La evolución de los programas y políticas de nutrición y alimentación en México es producto de un proceso de aprendizaje de las experiencias en programas y ac- ciones realizadas tanto en el país como en otros países. Asimismo, han sido utilizados por el Estado para contener la movilización social, y han sido transformados para disminuir el gasto social en los programas de ajuste estructural. ${ }^{2,3,25}$ Actualmente se sabe mucho más acerca de las causas de desnutrición, las acciones que se pueden llevar a cabo para mejorar el estado nutricional y los efectos que dichas intervenciones tienen en la población, y esto se refleja en los programas y políticas implementados mundialmente (por ejemplo, el énfasis ha cambiado de aumento de peso a aumento de talla para la edad como indicador de me- 
jora en el estado nutricional infantil, y se ha entendido que las intervenciones son más efectivas cuando incluyen componentes educativos y otras medidas integrales):;6 también se han desarrollado más y mejores métodos y técnicas para realizar evaluaciones y tomar mejores decisiones. ${ }^{27}$ El debate sobre alimentación y nutrición ha sido creciente; en la actualidad se cuenta con información útil en el país para comprender mejor las necesidades de salud y el funcionamiento de los programas ${ }^{28}$ La investigación en nutrición aplicada ha aumentado considerablemente, y legislativamente se han conducido consultas públicas alrededor de esta problemática $^{29}$ y se ha añadido, recientemente, una modificación a la Constitución en torno al derecho a la alimentación. ${ }^{30}$

Uno de los retos más importantes que enfrentan las políticas de salud y nutrición es el de llevar a cabo acciones con recursos limitados y que respondan a las necesidades locales. Las políticas de ajuste estructural asumidas por los países en desarrollo en momentos de crisis, generalmente tienen por objeto reducir el gasto gubernamental y, consecuentemente, el déficit fiscal para favorecer el crecimiento económico nacional y acceder a créditos internacionales. Entre estas políticas figura una disminución en la participación del Estado en subsidios, lo que en general conduce a un cambio a programas selectivos de salud y nutrición dirigidos a grupos vulnerables con intervenciones puntuales de impacto comprobado (inmunizaciones, tratamientos colectivos contra los parásitos, megadosis de vitamina A, etcétera). Esta serie de medidas han sido criticadas ya que, se considera, tienen una fuerte influencia de agencias financieras supranacionales, y proponen intervenciones mínimas, de bajo precio y elevada eficacia para mejorar indicadores de nivel de vida frente a un panorama complejo donde el desarrollo, el medio ambiente, la cultura, las situaciones geográficas y los problemas administrativos juegan un papel importante que estos indicadores no siempre reflejan. ${ }^{31,32} \mathrm{En}$ su último reporte estratégico de nutrición, salud y población, el Banco Mundial habla de buscar un papel más balanceado de los gobiernos en estos sectores; es decir, en lugar de que el Estado sea predominantemente proveedor, debería aumentar sus actividades en legislación, regulación, información y financiamiento. También propone un cambio en el abordaje de la salud, donde se busque sobre todo un manejo integral de la salud infantil y mejorar la prevención de las enfermedades mediante cambios de conducta, promoviendo la participación de la comunidad y con mayor atención al contexto, ${ }^{33}$ tratando de responder a las críticas que se han hecho de sus políticas. ${ }^{31,34}$
El panorama es alentador; en el mundo y en la nación se observa una tendencia positiva en el estado nutricional, como se refleja en el último informe del estado mundial de la nutrición (en diversos indicadores que van desde una reducción en las deficiencias por micronutrimentos hasta el desmedro $)^{35}$ y en otros reportes que abordan el impacto de la suplementación con micronutrimentos y enriquecimiento de alimentos. ${ }^{21} \mathrm{El}$ entendimiento de las interrelaciones entre los factores ha permitido que se lleven a cabo programas integrales que abordan de forma simultánea educación, salud, alimentación y desarrollo. La necesidad de aglutinar los esfuerzos de los diferentes actores relacionados con el problema alimentario se reconoce como de alta prioridad; el diseño de un programa integral con participación de diversos sectores permite lograr sinergias importantes en los resultados, evitar la duplicación de actividades y ahorrar recursos añadiendo, sin embargo, complejidad a su implementación. ${ }^{36-38}$ Entre los diversos sectores está también contemplada la propia comunidad; la participación de los beneficiarios de los programas se vislumbra como un elemento para la realización de los mismos; es deseable que esta participación sea activa para que les permita involucrarse en diferentes áreas de los programas como planeación, monitoreo y evaluación, así como el aumento de su capacidad para generar propuestas que busquen resolver los problemas que se detecten..$^{39}$

A pesar de la evolución que han presentado las políticas y programas de alimentación y nutrición en México, la desnutrición y las enfermedades asociadas con deficiencias de ciertos nutrimentos persisten, $\mathrm{y}$ nuevas enfermedades relacionadas con la alimentación que antes no representaban problema alguno en nuestro país, como la obesidad, han tomado rápidamente relevancia como problema de salud pública. ${ }^{28}$ Por ello, la importancia de esta área, a la luz del conocimiento actual, es determinante para el desarrollo nacional.

Adoptar una perspectiva de administración, en lugar de una de planificación para la implementación de programas, ${ }^{40}$ es una de las propuestas alternativas a las tradicionalmente verticales y centrales planteadas por el Banco Mundial en los setenta y que poco a poco se ha ido incorporando a los diseños de programas. ${ }^{41}$ Esta puede presentar ventajas, por diversas razones, como activar el compromiso político, cultivar y sostener la voluntad necesaria para apoyar programas de nutrición, relacionar el diseño con las capacidades organizativas y la realidad ambiental, coordinar las acciones, generar entendimiento y confianza, además de promover participación de la sociedad y ser el puente entre las estrategias, estructuras y proce- 
sos, conciliando y adaptando esta propuesta cuando surgen incongruencias. ${ }^{41}$ La necesidad de programas que respondan a las prioridades que se presentan en el nivel local demandan que éstos sean lo suficientemente flexibles para lograr así un funcionamiento adecuado y mejores resultados. Una evaluación continua de estos programas, así como la documentación sistemática de estas experiencias, se convierte en fuente importante de retroalimentación que se podría traducir en programas con mejor funcionamiento y consecuentes beneficios para la población.

Asimismo, desarrollar programas desde el punto de vista de la administración plantea el reto de mantener a los prestadores de servicios capacitados, sensibles a la necesidad de los grupos que se verán beneficiados, y con un entendimiento claro de las prioridades, para lo cual se requiere una política alimentaria y nutricional explícita, lo cual, como efecto adicional, fomentaría la permanencia de ésta más allá de los periodos sexenales o de otros cambios gubernamentales. Este último punto representa una de las principales preocupaciones de los beneficiarios de los programas y sus ejecutadores, así como uno de los principales puntos de escepticismo entre académicos y políticos.

Por último, vale la pena reflexionar en el hecho de que una parte importante del conocimiento y experiencia en implementación y planeación no es exclusiva de los programas alimentarios y de nutrición; ésta ha sido descrita y analizada a fondo en diversas áreas de la salud y de las políticas públicas, ${ }^{9,42,43}$ por ello, es importante revisar y aprovechar información de áreas convergentes que enfrentan problemas similares. ${ }^{38}$ En este sentido, vale la pena procurar mayor integralidad en la planificación e investigación en el campo de la nutrición y alimentación, con áreas del desarrollo como salud, educación y desarrollo sustentable, entre otras.

\section{Agradecimientos}

Agradecemos a los doctores Beatrice L. Rogers, Reynaldo Martorel, Jean Pierre Habitch y Gretel Pelto por los conceptos vertidos como profesores invitados de nuestro curso de Programas y Políticas de Alimentación, realizado en el Instituto Nacional de Salud Pública durante el verano de 1999, así como al doctor Víctor Ríos Cortázar, por los valiosos comentarios aportados a un borrador preliminar de este documento; finalmente, a la señorita Yadira González Orihuela, por su apoyo secretarial.

\section{Referencias}

1. Del Bajío A. Crisis alimentarias y subsistencias populares en México. México, D.F.: Leche Industrializada Conasupo, 1987.

2. Fox J.The politics of food in Mexico: State power and social mobilization. En: Buttel Frederick H, ed. Food Systems and A grarian Change. Ithaca: Cornel University Press, 1992.

3. Brachet-Márquez V. Political change and the welfare state:The case of health and food policies in Mexico (1970-1993). World Dev 1994: 1295-1312.

4. Chávez A, De C hávez M, Roldán A, Bermejo S,Avila A, Madrigal H.The Food and $N$ utrition Situation in Mexico:A food consumption, nutritional status and applied programs tendencies report from 1960 to 1990. México, D.F.: Editorial Pax México, 1996.

5. Lustig N . Food subsidy programs in Mexico. W ashington, D.C.: International Food Policy Research Institute, 1986.

6. Spalding R. El SistemaA limentario Mexicano (SAM): ascenso y decadencia. Estudios Sociológicos 1985;3(8):315-349.

7.Arteaga-Pérez J. El SistemaA Alimentario Mexicano (SAM): una perspectiva política. Estudios Sociológicos 1985;3(8):297-313.

8. Kennedy E. Alternatives to consumer-oriented food subsidies for achieving nutritional objectives. En: Pinstrup-Andersen P, ed. Food Subsidies in Developing Countries: Costs, benefits and policy options. Baltimore:The Johns Hopkins University Press, 1988:147-158.

9.W arwick D. Bitter Pills: Population policies and their implementation in eight developing countries. C ambrige, England:C ambridge U niversity Press, 1982.

10. Lustig N . Fiscal cost and welfare effects of the maize subsidy in Mexico. En: Pinstrup-Andersen P, ed. Food Subsidies in D eveloping Countries: Costs, benefits and policy options. Baltimore:The Johns Hopkins University Press, 1988:227-288.

11. Comisión Nacional de Alimentación (Conal). Seminario sobre criterios de seguimiento y evaluación de programas alimentarios y nutricionales. Memoria. Taller. México, D.F.: Conal-Solidaridad, 1992:45-49,67-68. 12. Barker J. The Liconsa Milk coupon program in Mexico (C ase study $\mathrm{XVI}$ ). En: From Platitudes to practice:Targeting social programs in Latin A merica.W ashington, D.C .: H uman Resources Division,Technical D epartment, Latin A merica and the Caribbean Region, The W orld Bank, 1992.

13. Rivera-D ommarco J. Editorial. Salud Publica Mex 1998;40(4):307-308. 14. Robles-Sardín A,Astiazarán-G arcía H, D ávalos-N avarro R, Q uihui-C ota $L, C$ abrera-Pacheco R, Valencia M. Efecto de la suplementación con una dosis masiva de vitamina A en niños de 6 a 36 meses de edad. Salud Publica Mex 1998:40(4):309-315.

15. Algunos aspectos sobre la atención a la niñez en el medio rural mexicano. México, D.F.: Comisión $\mathrm{N}$ acional para el Año Internacional del $\mathrm{N}$ iño, 1979.

16. Sistema $\mathrm{N}$ acional para el D esarrollo Integral de la Familia. El paquete de detección-atención. Sus elementos básicos. México, D.F.: Instituto $\mathrm{Na}$ cional de la Nutrición Salvador Zubirán, Subdirección General de Nutrición de Comunidad, 1991.

17. Programa Nacional de Alimentación y Nutrición familiar (Pronal). México, D.F.: Gobierno Federal de los Estados Unidos Mexicanos, 1995. 18. Ríos E, Martínez-Salgado H. Estrategias y recomendaciones para mejorar la nutrición de niños y mujeres en México. México, D.F.: Instituto $\mathrm{N}$ acional de N utrición Salvador Zubirán, Instituto $N$ acional de Salud Pública, 1994.

19. Programa de Educación, Salud y A limentación. D ocumento para la 2 da. Reunión de Inducción al Componente de Salud del Progresa. México, D.F.: Conprogresa, 1998. 
20. Coady D.A preliminary process evaluation of the Education, Health and N utrition Program (Progresa) of Mexico. W ashington, D.C.: International Food Policy Research Institute, 1999.

21. World Bank. Enriching lives. 0 vercoming Vitamin and Mineral Malnutrition in Developing Countries. Development in practice. Washington, D.C.:The W orld Bank, 1994.

22. Rosado J, Bourges H, Saint-Martín B. D eficiencia de vitaminas y minerales en Mexico. Una revisión crítica del estado de la información: I. D eficiencia de minerales. Salud Publica Mex 1995;37:130-139.

23. Maisterrena J,Tovar E, C hávez A. Bocio endémico en México: influencia de los factores nutricionales. Gac Med Mex 1964; 94:1123-1132.

24. Rosado J, Camacho-Solís R, Bourges H. Adición de vitaminas y minerales a harinas de maíz y de trigo en México. Salud Publica Mex 1999; 41(2):130-137.

25.W ilson J. Bureaucracy:W hat government agencies do and why they do it. Boston (MA): Basic Books, Inc./Harper Collins Publishers, 1989.

26. United N ations International Children's Emergency Fund (UN ICEF). Estado Mundial de la Infancia. N ueva York (N Y): UN IC EF, 1998.

27. Posavac E, Carey R. Program evaluation: Methods and case studies. $\mathrm{N}$ ueva Jersey (N J): Prentice Hall, 1990.

28. Encuesta $\mathrm{N}$ acional de Nutrición 1999. Cuernavaca, Mor.: Instituto N acional de Salud Pública, Secretaría de Salud - IN EGI, 2000.

29. Honorable Cámara de Diputados LI.Audiencias públicas sobre el problema de la alimentación en México, «D r. Salvador Zubirán». México D.F.: Comisión de distribución y manejo de bienes de consumo y servicio de la camara de diputados del H. Congreso de la Unión. LV Legislatura, 1993. 30. Rosales-O rtega S, Q uiroz-Ramírez C, ed. El derecho constitucional a la alimentación. México,D.F.:LV Legislatura, Cámara de Diputados.Comisión de Distribución y Manejo de Bienes de Consumo y Servicios, 1994.

31. López-A rellano 0. La selectividad en la política de salud. En: Laurell C, ed. $N$ uevas tendencias y alternativas en el sector salud. México D.F.:Universidad Autónoma Metropolitana-Xochimilco, Fundación Frederich Hebert, 1995.
32. Levinson J. Incorporating nutrition into bank-assisted social funds. W ashington, D.C.:TheW orld Bank, Human Resources D evelopment and 0 perations Policy, 1993.

33.W orld Bank. Health, N utrition and Population. W ashington, D.C.:The World Bank Group, Human Development N etwork, 1997.

34. Field J. Multisectorial nutrition planning: A post-mortem. Food Policy 1987:15-28.

35.ACC/SCN . Fourth Report on the W orld N utrition Situation: $N$ utrition throughout the life cicle. W ashington, DC.: United Nations, 2000.

36. Petit M. D eterminants of food policies:An atempt to understand government behavior. En: Pinstrup-Andersen P, ed. The Political Economy of Food and N utrition Policies. Londres:The Johns H opkins University Press, 1993:22-33.

37. Florentino R,A dorna C, Solon F. Interface problems between nutrition policy and its implementation:The Philipine $\mathrm{C}$ ase Study. En:Scimshaw $\mathrm{N}$ aW MB, ed. N utrition Policy Implementation: Issues and Experience. N ueva York (N Y): Plenum Press, 1982:247-268.

38. Field J. Implementing $N$ utrition Programs: Lessons from an unheeded Literature.Ann Rev Nutr 1984;5:143-172.

39. Chambers R.W hose reality counts? Putting the first last. Londres: Intermediate Technology Publications, 1997.

40. Field J.The importance of context: N utrition planning and development reconsidered. En: McLaren D, ed. N utrition in the community. N uevaYork (N Y): John W iley and Sons, 1983:61-78.

41. Field J. From nutrition planning to nutrition management:The politics of action. En: Pinstrup-Andersen P, ed. The Political Economy of Food and N utrition Policies. Londres:The Johns H opkins U niversity Press, 1993:173192.

42. C aiden $N$, W ildavskyA. Planning and budgeting in poor countries. N ueva Brunswick (N J): Transaction Books, 1985.

43. Pressman J,W ildavsky A. Implementation. Berkeley (CA): University of California Press, 1984. 